\title{
Strong Feller solutions to SPDE's are strong Feller in the weak topology
}

\author{
by \\ Bohdan Maslowski and Jan Seidler (Praha)
}

\begin{abstract}
For a wide class of Markov processes on a Hilbert space $H$, defined by semilinear stochastic partial differential equations, we show that their transition semigroups map bounded Borel functions to functions weakly continuous on bounded sets, provided they map bounded Borel functions into functions continuous in the norm topology. In particular, an Ornstein-Uhlenbeck process in $H$ is strong Feller in the norm topology if and only if it is strong Feller in the bounded weak topology. As a consequence, it is possible to strengthen results on the long-time behaviour of strongly Feller processes on $H$ : we extend the embedded Markov chains method of constructing a $\sigma$-finite invariant measure by replacing recurrent compact sets with recurrent balls, and in the transient case we prove that the last exit time from every weakly compact set is finite almost surely.
\end{abstract}

0. Introduction. Regularity properties of the transition semigroup $\left(P_{t}\right)$ of a Markov process play an important rôle in studying the long-time behaviour of the process. In particular, if the semigroup is strong Feller (i.e., $P_{t} \varphi$ is a continuous function for each bounded Borel function $\varphi$ and each $t>0)$ then refined tools from ergodic theory are applicable and a rather complete description of the asymptotic behaviour of the process in terms of invariant measures and recurrence properties is available. For Markov processes defined by stochastic differential equations in $\mathbb{R}^{d}$ a proof of the strong Feller property may be based on properties of the associated Kolmogorov equations and the theory of linear parabolic equations, provided the diffusion matrix is nondegenerate. As far as stochastic partial differential equations, defining Markov processes on an infinite-dimensional Hilbert space $H$, are concerned, such analytical methods are not easily applicable and the proofs become involved. (We refer the reader to the basic monograph [1] for all notions concerning stochastic partial differential equations we shall use below, and to [2] for a thorough discussion of the strong Feller property of infinite-dimensional diffusions.)

2000 Mathematics Subject Classification: 60J35, 60H15.

This research has been supported by the GA ČR Grant 201/98/1454. 
In a recent paper [12] we proposed an alternative probabilistic method, giving in some cases a straightforward proof of the strong Feller property. Moreover, it has been noticed in [13] that a closely related argument may be used to establish another strengthening of the classical Feller property, namely, to show that $P_{t} \varphi$ is a sequentially weakly continuous function whenever $\varphi$ is (sequentially) weakly continuous.

In the present note we use these results to make a step further: we find a (fairly wide) class of infinite-dimensional diffusions for which the strong Feller property implies that the functions $P_{t} \psi, \psi$ bounded Borel, are in fact sequentially weakly continuous. For us, the results obtained were surprising: it has been indicated above that the strong Feller property is rather "rare" and not easy to establish, so we had not expected that it might be automatically equivalent to a much stronger smoothing property in many nontrivial examples.

Let us call a transition semigroup $\left(P_{t}\right)$ bw-strong Feller if $P_{t} \psi$ is sequentially weakly continuous for all $t>0$ and each bounded Borel function $\psi$ (this terminology is explained below). In Theorem 1, we shall give a self-contained proof, based on the Cameron-Martin formula, that OrnsteinUhlenbeck processes in $H$ defined by linear equations

$$
d X=A X d t+\Sigma d W
$$

are $b w$-strong Feller if and only if they are strong Feller. In Theorem 2 we extend the result on equivalence of strong Feller and $b w$-strong Feller properties to semilinear equations

$$
d X=(A X+f(X)) d t+\sigma(X) d W
$$

with a (cylindrical) Wiener process $W$, an operator $A$ generating a compact semigroup on $H$ and with Lipschitz continuous nonlinear terms $f$ and $\sigma$. Using the Girsanov theorem we then show in Theorem 3 how to relax conditions on the drift $f$ without violating the $b w$-strong Feller property.

Besides providing an insight into the structure of strong Feller diffusions, the $b w$-strong Feller property has applications to their ergodic theory. The long-time behaviour of strong Feller irreducible Markov processes on Polish spaces is subject to a well known dichotomy: either there exists a recurrent compact set, and then the process is Harris recurrent (in particular, it has an essentially unique $\sigma$-finite invariant measure), or the process is transient and there is no finite invariant measure (see e.g. [16] and references therein). Although some results on existence of recurrent compact sets are available (cf., for example, a recent paper [10]), it is usually much more promising to try to find recurrent balls using suitable Lyapunov criteria. So it is of some interest that for $b w$-strong Feller processes the results on dichotomy remain valid even if recurrent balls are considered instead of recurrent compact sets. 
In particular, we may use embedded Markov chains with a state space only weakly compact to construct a $\sigma$-finite invariant measure for a recurrent process. A precise statement may be found in Theorem 4. In the transient case it was shown in [16] that existence of a "sufficiently large" compact set which is not recurrent implies that the last exit time from every compact set is almost surely finite. This result is not completely satisfactory, since for processes on infinite-dimensional Hilbert spaces it does not yield that

$$
\lim _{t \rightarrow \infty}\left|X_{t}\right|=\infty
$$

However, the property $(0.3)$ is generally employed to characterize the transience of diffusions in $\mathbb{R}^{n}$. In Theorem 5 we shall prove that $(0.3)$ is also valid in infinite-dimensional Hilbert spaces for transient $b w$-strong Feller processes.

In many particular situations, for the strong Feller property to hold the nondegeneracy of the noise term is necessary, and the covariance operator of the driving Wiener process cannot be nuclear, which precludes using Lyapunov functions like $|\cdot|^{p}$ for some $p>0$. Hence, it is shown in Example 2 that recurrence of balls may sometimes be established by employing quadratic forms $x \mapsto\langle R x, x\rangle$, with a nuclear operator $R$, as Lyapunov functions.

In what follows we shall need some results on sequentially weakly continuous functions which we collect here. Define the bounded weak topology $b w$ on a real separable Hilbert space $(H,|\cdot|)$ as the finest topology on $H$ that coincides with the weak topology of $H$ on every norm bounded subset of $H$, i.e., a set $F \subset H$ is $b w$-closed if and only if $F \cap U$ is weakly closed in $U$ for any bounded set $U$ (equivalently, for any ball $U$ ). Note that, in particular, all weakly compact sets are $b w$-compact. The topology $b w$ is compatible with the duality $\left\langle H, H^{*}\right\rangle$. (See e.g. [3], Section II.5, or [14], Section 2.7, for further information on the bounded weak topology.) Let us denote by $\mathscr{B}$ the Borel $\sigma$-algebra on $(H,|\cdot|)$. Owing to separability of $H, \mathscr{B}$ coincides with the weakly Borel sets, hence obviously the Borel $\sigma$-algebra over $(H, b w)$ also equals $\mathscr{B}$. Consequently, $(H, b w)$ is a Radon space.

Finally, let us note that $\varphi: H \rightarrow \mathbb{R}$ is bw-continuous if and only if $\varphi$ is sequentially weakly continuous. Indeed, set $K_{n}=\{x \in H:|x| \leq n\}$, $n \in \mathbb{N}$, and note that $\left(K_{n}, b w\right)$ are metrizable compact spaces. If $\varphi$ is $b w$ continuous and $x_{j} \rightarrow x$ weakly, then find $n \geq 1$ such that $x_{j}, x \in K_{n}$; the weak continuity of $\left.\varphi\right|_{K_{n}}$ implies $\varphi\left(x_{j}\right) \rightarrow \varphi(x)$. In the opposite direction, let $\varphi$ be sequentially weakly continuous. Then $\left.\varphi\right|_{K_{n}}$ is weakly continuous on any $K_{n}$ by metrizability of the weak topology on $K_{n}$. If $U \subset \mathbb{R}$ is an arbitrary open set, then $\varphi^{-1}(U) \cap K_{n}=\left(\left.\varphi\right|_{K_{n}}\right)^{-1}(U)$ is weakly open in $K_{n}$, so $\varphi^{-1}(U)$ is bw-open and bw-continuity of $\varphi$ follows.

We close this section with introducing some notation. We denote by $\mathbf{1}_{\Lambda}$ the indicator of a set $\Lambda$ and by $\mathrm{b} \mathscr{B}$ the space of all bounded real 
$\mathscr{B}$-measurable functions on $H$. We use $\mathscr{C}_{\mathrm{b}}(H)$ to denote the space of all bounded real continuous functions on $(H,|\cdot|)$, and $\mathscr{C}_{\mathrm{b}}(H, b w)$ for its subspace of bounded $b w$-continuous functions. If $X, Y$ are Hilbert spaces, then $\mathscr{L}(X, Y)$ denotes the space of all bounded linear operators from $X$ to $Y$, $\mathscr{L}(X)=\mathscr{L}(X, X)$, and $I \in \mathscr{L}(X)$ is the identity operator. $\|B\|_{\mathrm{HS}}$ denotes the Hilbert-Schmidt norm of an operator $B \in \mathscr{L}(X, Y)$. By $\stackrel{q}{\longrightarrow}$ we denote the convergence in $q$-measure, and $\mathscr{N}(m, Q)$ stands for a Gaussian measure with mean $m$ and covariance operator $Q$.

Let $P=\left(P_{t}(x, \cdot)\right)_{t \geq 0}$ be a transition function (a semigroup of Markov kernels) on $(H, \mathscr{B})$; we shall use the same symbol $\left(P_{t}\right)$ for the corresponding transition semigroup on $\mathrm{b} \mathscr{B}$. Finally, recall that $P$ is strong Feller if $P_{t}(\cdot, A) \in \mathscr{C}_{\mathrm{b}}(H)$, and $b w$-strong Feller if $P_{t}(\cdot, A) \in \mathscr{C}_{\mathrm{b}}(H, b w)$, for all $t>0$ and $A \in \mathscr{B}$.

1. Results. Let $H$ be a separable Hilbert space, $W$ a standard cylindrical Wiener process in a real separable Hilbert space $\Upsilon$, defined on a probability space $(\Omega, \mathscr{F}, \boldsymbol{P})$, and let $\Sigma \in \mathscr{L}(\Upsilon, H)$. The norm and the inner product in both $H$ and $\Upsilon$ will be denoted by $|\cdot|,\langle\cdot, \cdot\rangle$, respectively. Assume that $A: \operatorname{Dom}(A) \rightarrow H$ is an infinitesimal generator of a $C_{0}$-semigroup $\left(e^{A t}\right)$ on $H$. First, we shall consider an Ornstein-Uhlenbeck process in $H$, defined by the stochastic differential equation

$$
d Z=A Z d t+\Sigma d W .
$$

It is well known that if

$$
\int_{0}^{T}\left\|e^{A t} \Sigma\right\|_{\mathrm{HS}}^{2} d s<\infty \quad \text { for a } T>0,
$$

then for every $y \in H$ there exists a unique mild solution $Z^{y}$ to (1.1) with the initial condition $Z^{y}(0)=y$, given by the variation of constants formula

$$
Z^{y}(t)=e^{A t} y+\int_{0}^{t} e^{A(t-s)} \Sigma d W(s), \quad t \geq 0 .
$$

Accordingly, the equation (1.1) defines a Markov process in $H$ with transition probability $R$ satisfying

$$
R_{t}(x, \Gamma) \equiv \boldsymbol{P}\left\{Z^{x}(t) \in \Gamma\right\}=\mathscr{N}\left(e^{A t} x, Q_{t}\right)(\Gamma), \quad t>0, x \in H, \Gamma \in \mathscr{B},
$$

where

$$
Q_{t}=\int_{0}^{t} e^{A r} \Sigma \Sigma^{*} e^{A^{*} r} d r, \quad t \geq 0 .
$$

Note that $Q_{t}$ is a nonnegative nuclear operator by (1.2). We aim at proving the following result on regularity of $R$ : 
Theorem 1. Assume (1.2), let $R$ be the transition function defined by the equation (1.1). Then the following statements are equivalent:

(a) For all $t>0$,

$$
\operatorname{Rng} e^{A t} \subseteq \operatorname{Rng} Q_{t}^{1 / 2} .
$$

(b) $R$ is strong Feller.

(c) $R$ is bw-strong Feller.

(d) For every $t>0$ and any $y_{n}, y_{0} \in H$ such that $y_{n} \rightarrow y_{0}$ weakly,

$$
\lim _{n \rightarrow \infty}\left\|\mid R_{t}\left(y_{n}, \cdot\right)-R_{t}\left(y_{0}, \cdot\right)\right\|=0
$$

$\||\|| \mid$ denoting the total variation norm on the space of all bounded signed Borel measures on $H$.

It is well known that (a) is equivalent to (b) (see e.g. [1], Section 9.4.1), so it remains to check only that (d) follows from (a), the proof of this implication being deferred to Section 2 .

REMARK 1. The assertion (d) of Theorem 1 says, in other words, that $R$ as a semigroup of Markov kernels on $((H, b w), \mathscr{B})$ is strong Feller in the strict sense (in the terminology of [8], Definition (B)) or ultra-Feller (in the terminology of [4], Définition IX.8). G. Mokobodzki showed that a transition semigroup is strong Feller if and only if it is ultra-Feller. A proof of this result for metric state spaces may be found e.g. in [4], Théorème IX.18; with minor modifications, the same argument also applies to processes on $(H, b w)$. We do not need this general result to prove Theorem 1, but it may be used to strengthen Theorems 2 and 3 below in an obvious way.

Now we turn to the properties of a transition function $P$ defined by a semilinear equation

$$
d X=(A X+f(X)) d t+\sigma(X) d W .
$$

Let us start with a simple observation: the semigroup $\left(P_{t}\right)$ is bw-strong Feller provided it is strong Feller and

$$
P_{t}\left(\mathscr{C}_{\mathrm{b}}(H)\right) \subseteq \mathscr{C}_{\mathrm{b}}(H, b w) \quad \text { for all } t>0 .
$$

Indeed, let $\varphi \in \mathrm{b} \mathscr{B}$ and $t>0$ be arbitrary; then $P_{t / 2} \varphi \in \mathscr{C}_{\mathrm{b}}(H)$ by the strong Feller property, so $P_{t} \varphi=P_{t / 2}\left(P_{t / 2} \varphi\right) \in \mathscr{C}_{\mathrm{b}}(H, b w)$ by (1.6). However, in [13] simple sufficient conditions for (1.6) to hold have been found. Let us recall the basic result ([13], Theorem 2.2) concerning equations with an operator $A$ generating a compact semigroup and with $f, \sigma$ Lipschitz continuous: Suppose that $A: \operatorname{Dom}(A) \rightarrow H$ generates a $C_{0}$-semigroup on $H$ and $W$ is a (possibly cylindrical) Wiener process in $\Upsilon$ with a covariance operator $Q$, $Q \in \mathscr{L}(\Upsilon)$ a self-adjoint nonnegative operator. Let the range $\operatorname{Rng} Q^{1 / 2}$ be endowed with its natural Hilbert space structure (cf. [1], Section 4.2). 
Theorem 2. Assume that $f: H \rightarrow H$ and $\sigma: H \rightarrow \mathscr{L}\left(\operatorname{Rng} Q^{1 / 2}, H\right)$ are Borel functions and there exist a constant $K<\infty$ and a function $k \in$ $L_{\mathrm{loc}}^{1}([0, \infty[), k \geq 0$ such that

$$
\begin{aligned}
& |f(x)-f(y)| \leq K|x-y|, \\
& \left\|e^{A t} \sigma(x) Q^{1 / 2}\right\|_{\mathrm{HS}}^{2} \leq k(t)\left(1+|x|^{2}\right), \\
& \left\|e^{A t}[\sigma(x)-\sigma(y)] Q^{1 / 2}\right\|_{\mathrm{HS}}^{2} \leq k(t)|x-y|^{2}
\end{aligned}
$$

for all $t \geq 0, x, y \in H$. Let the semigroup $\left(e^{A t}\right)$ be compact. Then the transition semigroup $\left(P_{t}\right)$ defined by equation (1.5) satisfies (1.6). Therefore, $\left(P_{t}\right)$ is strong Feller if and only if it is bw-strong Feller.

Remark 2. Theorem 1 clearly follows from Theorem 2 and the Mokobodzki theorem cited in Remark 1. However, we have found Theorem 1 being worth a self-contained and rather elementary direct proof.

It has been shown in [12] that, under suitable hypotheses, a Markov process related to a strong Feller Markov process by the Girsanov transform is again strong Feller; this procedure makes it possible to considerably relax assumptions on the drift of (1.5) when proving the strong Feller property. A similar statement holds true for the bw-strong Feller property: We shall consider a pair of equations

$$
\begin{aligned}
d Z & =(A Z+g(Z)) d t+\sigma(Z) Q^{1 / 2} d W, \\
d X & =(A X+f(X)) d t+\sigma(X) Q^{1 / 2} d W,
\end{aligned}
$$

in which $A: \operatorname{Dom}(A) \rightarrow H$ is again a generator of a $C_{0^{-}}$-semigroup on $H$, $W$ a standard cylindrical Wiener process on $\Upsilon, Q \in \mathscr{L}(\Upsilon)$ a self-adjoint nonnegative operator, and $f, g: H \rightarrow H$ and $\sigma: H \rightarrow \mathscr{L}\left(\operatorname{Rng} Q^{1 / 2}, H\right)$ are Borel mappings. We shall assume

(A) (1) There exists a probability space $(\Omega, \mathscr{F}, \boldsymbol{P})$ carrying a standard cylindrical Wiener process $W$ on $\Upsilon$ and, for any $y \in H$, a mild solution $Z^{y}$ to (1.7) with continuous trajectories satisfying $Z^{y}(0)$ $=y$.

(2) For any $y \in H$ there exists a martingale solution $\left(\left(\Xi_{y}, \mathscr{J}^{y}, q^{y}\right)\right.$, $\left.\left(\mathscr{J}_{t}^{y}\right), W^{y}, X^{y}\right)$ to $(1.8)$ with continuous trajectories and with $X^{y}(0)=y$.

(3) Uniqueness in law holds for both (1.7) and (1.8).

(We recall that martingale solutions are discussed in [1], Chapter 8; in what follows we do not need any particular properties of them, only the existence of a transition function is relevant.) From (A) it follows that the equations (1.7), (1.8) define Markov processes. Let $R, P$ be their respective transition 
functions, that is,

$$
\begin{aligned}
R_{t} \varphi(y)=\boldsymbol{E} \varphi\left(Z^{y}(t)\right), \quad P_{t} \varphi(y)= & \int_{\Xi_{y}} \varphi\left(X^{y}(t)\right) d q^{y}, \\
& t \geq 0, y \in H, \varphi \in \mathrm{b} \mathscr{B} .
\end{aligned}
$$

We shall prove the following result on the bw-strong Feller property.

THEOREM 3. Let the assumption (A) be satisfied and assume that there exists a Borel function $u: H \rightarrow \Upsilon$ such that $f=g+\sigma Q^{1 / 2} u$. Set

$$
U(y, t)=\exp \left(\int_{0}^{t}\left\langle u\left(Z^{y}(s)\right), \cdot\right\rangle d W(s)-\frac{1}{2} \int_{0}^{t}\left|u\left(Z^{y}(s)\right)\right|^{2} d s\right)
$$

for $t \geq 0, y \in H$. Suppose that

(a) $\boldsymbol{E} U(y, t)=1$ for every $t \geq 0$ and $y \in H$,

and either

(b) the transition function $R$ is bw-strong Feller,

(c) the transition function $P$ is bw-Feller, i.e., for each $t>0$,

$$
P_{t}\left(\mathscr{C}_{\mathrm{b}}(H, b w)\right) \subseteq \mathscr{C}_{\mathrm{b}}(H, b w),
$$

(d) the set $\left\{U\left(y_{n}, t\right): n \geq 1\right\}$ is uniformly integrable for any $t \geq 0$ and any weakly convergent sequence $\left\{y_{n}\right\}$ in $H$,

or

(e) the transition function $P$ is strong Feller,

(f) for every $t>0$, all $y_{n}, y \in H$ such that $y_{n} \rightarrow y$ weakly, and any $\varphi \in \mathscr{C}_{\mathrm{b}}(H)$

$$
\begin{gathered}
U\left(y_{n}, t\right) \underset{n \rightarrow \infty}{\stackrel{P}{\longrightarrow}} U(y, t), \\
\varphi\left(Z^{y_{n}}(t)\right) \underset{n \rightarrow \infty}{\stackrel{P}{\longrightarrow}} \varphi\left(Z^{y}(t)\right) .
\end{gathered}
$$

Then $P$ is bw-strong Feller.

Note that the assumption (A)(2) is satisfied provided (a) holds. Further, if $u$ is a continuous function of linear growth, then (1.9) follows from

$$
\lim _{n \rightarrow \infty} \int_{0}^{T} \boldsymbol{E}\left|Z^{y_{n}}(t)-Z^{y}(t)\right|^{2} d t=0
$$

for any $T>0, y_{n}, y \in H, y_{n} \rightarrow y$ weakly. The hypothesis (1.11) is easy to check in many particular situations (e.g., under the assumptions of Theorem 2, cf. [13]). Analogously, (1.10) is a consequence of

$$
\left|Z^{y_{n}}(t)-Z^{y}(t)\right| \underset{n \rightarrow \infty}{\stackrel{P}{\longrightarrow}} 0 .
$$


Finally, it is possible to omit the assumption (c), supposing instead that (1.9) and (1.10) are satisfied for every $\varphi \in \mathscr{C}_{\mathrm{b}}(H, b w)$ (this will be clear from the proof of Theorem 3, cf. also [12], Theorem 2.1).

The proof of Theorem 3 is a combination of the proofs of Theorem 2.1 in [12] (with minor modifications) and Theorem 2.3 in [13]; for the reader's convenience we sketch the simple argument in Section 2.

EXAMPLE 1. It might seem difficult to apply Theorem 3 in a straightforward manner since its assumptions refer to rather nontrivial properties of equations (1.7), (1.8), like the strong Feller or bw-Feller properties. Therefore, it is worth realizing that for many semilinear equations with an additive noise we may use Theorem 3 to obtain a self-contained proof (relying on Theorem 1 only) of the $b w$-strong Feller property. Let us consider the problem (1.8) with $\sigma=I$, that is, the equation

$$
d X=(A X+f(X)) d t+Q^{1 / 2} d W .
$$

Suppose that

$$
\operatorname{Tr} \int_{0}^{t} e^{A r} Q e^{A^{*} r} d r<\infty
$$

and

$$
\operatorname{Rng} e^{A t} \subseteq \operatorname{Rng}\left(\int_{0}^{t} e^{A r} Q e^{A^{*} r} d r\right)^{1 / 2}
$$

for all $t>0$, that $\operatorname{Rng} f \subseteq \operatorname{Rng} Q^{1 / 2}$, and that $Q^{-1 / 2} f: H \rightarrow H$ is a continuous function of a linear growth, where $Q^{-1 / 2}$ denotes the pseudoinverse. By Theorem 1, the Ornstein-Uhlenbeck process

$$
d Z=A Z d t+Q^{1 / 2} d W
$$

is bw-strong Feller, and with the choice $u=Q^{-1 / 2} f$ the assumption (a) of Theorem 3 is satisfied. The semigroup $\left(e^{A t}\right)$ is compact by (1.14), hence (1.11), (1.12) hold implying hypotheses (c), (d) of Theorem 3. If weak uniqueness holds for (1.13) we may use Theorem 3 to conclude that (1.13) defines a $b w$-strong Feller process.

The following two theorems are devoted to consequences of the $b w$-strong Feller property for the long-time behaviour of Markov processes. Assume that $\left(\Omega, \mathscr{F},\left(\mathscr{F}_{t}\right),\left(\theta_{t}\right), X, \boldsymbol{P}_{x}\right)$ is a homogeneous Markov process on $H$ with continuous trajectories (in the norm topology). Denote by $P$ its transition function and recall that $\left(\theta_{t}\right)$ are the shift operators. We say that a set $\Lambda \subseteq H$ is recurrent provided $\boldsymbol{P}_{x}\left\{\tau_{\Lambda}<\infty\right\}=1$ for all $x \in H$, where

$$
\tau_{\Lambda}=\inf \left\{t>0: X_{t} \in \Lambda\right\}
$$


is the first hitting time of $\Lambda$, and that $\Lambda$ is transient if

$$
\boldsymbol{P}_{x}\left\{\sup \left\{t \geq 0: X_{t} \in \Lambda\right\}<\infty\right\}=1
$$

for every $x \in H$. (As usual, we set $\inf \emptyset=+\infty, \sup \emptyset=0$.)

Theorem 4. Assume that $P$ is bw-strong Feller and that the measures $P_{t}(x, \cdot), t>0, x \in H$, are all equivalent. Let the filtration $\left(\mathscr{F}_{t}\right)$ be rightcontinuous and complete. Let there exist a recurrent weakly compact set $J$ in H. Then:

(a) All Borel sets $B \in \mathscr{B}$ such that $P_{1}(0, B)>0$ are recurrent.

(b) There exists a $\sigma$-finite invariant measure $\nu$ for $P$. If moreover

$$
\sup _{x \in J} \boldsymbol{E}_{x} \theta_{\delta} \tau_{J}<\infty
$$

for some $\delta>0$ then $\nu(H)<\infty$.

REMARK 3. (i) Obviously, due to the equivalence hypothesis we may replace the assumption $P_{1}(0, B)>0$ by $P_{r}(z, B)>0$ with arbitrary $r>0$, $z \in H$. We need the assumptions on the filtration only to know that the first hitting time of $B$ is a stopping time and these assumptions may be omitted if only closed sets $B$ are considered, which is sufficient for the construction of an invariant measure.

(ii) The measure $\nu$ is Radon in the sense that it is bw-locally finite (for each $x \in H$, there is a bw-open neighbourhood $V \ni x$ with $\nu(V)<\infty)$ and inner regular with respect to (norm) compact sets (for all $V \in \mathscr{B}$, $\nu(V)=\sup \{\nu(C): C \subseteq V, C$ compact $\})$.

(iii) A standard proof shows that if $P$ is $b w$-strong Feller and $b w$-irreducible (that is, $P_{t} \mathbf{1}_{V}>0$ on $H$ for all $t>0$ and every $V \neq \emptyset b w$-open in $H)$ then all measures $P_{t}(x, \cdot)$ are equivalent. Let us note that the transition semigroup $\left(P_{t}\right)$ considered in Example 1 is also irreducible. (For linear equations, irreducibility follows from the strong Feller property, and it is obviously preserved by the Girsanov transform.)

(iv) The hypothesis (1.15) has an obvious interpretation: note that $\eta+$ $\theta_{\eta} \tau_{J}$ is the first hitting time of $J$ after $\eta$ for any $\eta \in \mathbb{R}_{+}$.

Remark 4. Assume, in addition, that

$$
\begin{aligned}
& \text { For any } \varkappa>0 \text { and each } K \subseteq H \text { compact, } \\
& \qquad \lim _{t \rightarrow 0+} \sup _{y \in K} \boldsymbol{P}_{y}\left\{\sup _{0 \leq s \leq t}\left|X_{s}-y\right| \geq \varkappa\right\}=0 .
\end{aligned}
$$

Then all results on the long-time behaviour of recurrent processes contained in Theorem 2.2 and Corollary 2.3 of [16] (Harris recurrence, uniqueness of a $\sigma$-finite invariant measure, the ratio ergodic theorem) remain valid, with proofs essentially unchanged. (In their proofs, Theorem 4 now replaces Lemma 5.1 of [16].) The hypothesis (B) seems to be rather restrictive, but 
it is typically satisfied by diffusion processes defined by stochastic parabolic equations (Section 3 in [16] contains several related examples).

The proof of Theorem 4 is given in Section 2.

Further, let us consider the transient case.

Theorem 5. Suppose that (B) holds, $P$ is bw-strong Feller and the measures $P_{t}(x, \cdot), t>0, x \in H$, are all equivalent. Let there exist a (norm) compact set $K \subseteq H$ which is not recurrent but $P_{1}(0, K)>0$. Then all weakly compact subsets of $H$ are transient. In particular,

$$
\lim _{t \rightarrow \infty}\left|X_{t}\right|=+\infty \quad \boldsymbol{P}_{x} \text {-almost surely }
$$

for all $x \in H$.

Note that if there is a nonrecurrent ball $B$ in $H$ with $P_{1}(0, B)>0$ then we can find a compact set $K$ with the desired properties. (Indeed, the measure $P_{1}(0, \cdot)$ is Radon, hence $P_{1}(0, K)>0$ for some compact $K \subseteq B$ and, obviously, $K$ cannot be recurrent.)

Proposition 2.2 in [7] shows that under the assumptions of Theorem 5 transient sets $B_{n}, n \geq 1$, may be found such that $B_{n} \nearrow H$. A straightforward modification of the proof shows that all weak compact sets are transient; see Section 2 for some details.

Remark 5. Assume that (B) holds, the measures $P_{t}(x, \cdot), t>0, x \in H$, are equivalent and $P$ is $b w$-strong Feller. Then a standard proof (essentially due to Khas'minskiu) yields that

$$
\sup _{x \in H} \int_{0}^{\infty} P_{t}(x, C) d t<\infty
$$

for each nonrecurrent weakly compact set $C \subseteq H$; cf. e.g. the proof of Theorem 2.1 in [16].

This result implies that, under the same hypotheses, there always exists at least one locally finite subinvariant (excessive) measure $Q$ for $P$; consequently, the transition semigroup $\left(P_{t}\right)$ extends to a contraction semigroup on $L^{p}(H, Q)$ for all $1 \leq p<\infty$. Indeed, if a recurrent ball exists then we can even find a locally finite invariant measure according to Theorem 4 . In the opposite case, take a finite Borel measure $q$ on $H$ and set

$$
Q(\cdot)=\int_{0}^{\infty} \int_{H} P_{t}(x, \cdot) d q(x) d t .
$$

The measure $Q$ is subinvariant and $Q(B)<\infty$ for all balls $B$ in $H$ by (1.16); see e.g. [18] for a discussion of closely related results.

Finally, we shall address the problem of verifying the hypothesis (1.15) of Theorem 4 by means of Lyapunov functions techniques. 
ExAmple 2. Consider a stochastic evolution equation

$$
d X=(A X+f(X)) d t+\sigma(X) d W
$$

in $H$. Let $W$ be a standard cylindrical Wiener process in $\Upsilon$, and assume that $A: \operatorname{Dom}(A) \rightarrow H$ generates a $C_{0}$-semigroup $\left(e^{A t}\right)$ on $H$ such that $\left\|e^{A t}\right\|_{\mathscr{L}(H)} \leq M e^{-\omega t}$ for some $M \geq 1, \omega>0$ and all $t \geq 0$, and that

$$
\int_{0}^{T} t^{-\varrho}\left\|e^{A t}\right\|_{\mathrm{HS}}^{2} d t<\infty
$$

for some $\varrho>0, T>0$. Let $f: H \rightarrow H, \sigma: H \rightarrow \mathscr{L}(\Upsilon, H)$ be Lipschitz continuous functions. Let $\left(X, \boldsymbol{P}_{x}\right)$ be the Markov process solving (1.17); it is known that

$$
\sup _{0 \leq s \leq t} \boldsymbol{E}_{x}|X(s)|^{2} \leq C_{t}\left(1+|x|^{2}\right)
$$

for a constant $C_{t}<\infty$ depending only on $M, t$ and the constants $K_{f}, K_{\sigma}$ in the linear growth estimates

Set

$$
|f(x)| \leq K_{f}(1+|x|), \quad\|\sigma(x)\|_{\mathscr{L}(\Upsilon, H)} \leq K_{\sigma}(1+|x|), \quad x \in H .
$$

$$
R=2 \int_{0}^{\infty} e^{A t} e^{A^{*} t} d t .
$$

Then $R \in \mathscr{L}(H)$ is a self-adjoint nonnegative nuclear operator, and $\langle R x, A x\rangle$ $=-|x|^{2}$ for every $x \in \operatorname{Dom}(A)$. Denote by $B_{L}$ the closed ball of radius $L$ centered at 0 , and assume that there exist $\alpha<0$ and $L \in] 0, \infty[$ such that

$$
-|y|^{2}+\langle R y, f(y)\rangle+\frac{1}{2} \operatorname{Tr}\left(R \sigma(y) \sigma^{*}(y)\right) \leq \alpha \quad \text { for every } y \notin B_{L} .
$$

Then

$$
\boldsymbol{E}_{x} \tau_{\eta} \leq \eta+\frac{1}{2|\alpha|} C_{\eta}\|R\|_{\mathscr{L}(H)}\left(1+|x|^{2}\right) \quad \text { for all } x \in H,
$$

where $\tau_{\eta}$ denotes the first hitting time of $B_{L}$ after $\eta \geq 0$. Indeed, by [11], Proposition 1.5, we may apply the Itô formula to the process $\frac{1}{2}\langle R X, X\rangle$ to obtain

$$
\begin{aligned}
\frac{1}{2} \boldsymbol{E}_{x}\left\langle R X\left(\tau_{\eta}\right), X\left(\tau_{\eta}\right)\right\rangle-\frac{1}{2} \boldsymbol{E}_{x}\langle R X(\eta), X(\eta)\rangle \\
\quad=\boldsymbol{E}_{x} \int_{\eta}^{\tau_{\eta}}\left\{-|X(s)|^{2}+\langle R X(s), f(X(s))\rangle+\frac{1}{2} \operatorname{Tr}\left(R \sigma(X(s)) \sigma^{*}(X(s))\right)\right\} d s .
\end{aligned}
$$

Since $\left\langle R X\left(\tau_{\eta}\right), X\left(\tau_{\eta}\right)\right\rangle \geq 0$ and $X(s) \notin B_{L}$ for $\left.s \in\right] \eta, \tau_{\eta}[$, (1.19) implies

$$
-\frac{1}{2} \boldsymbol{E}_{x}\langle R X(\eta), X(\eta)\rangle \leq \alpha \boldsymbol{E}_{x}\left(\tau_{\eta}-\eta\right)
$$

so the desired estimate (1.20) follows from (1.18). 
Taking into account that

$$
\|R\|_{\mathscr{L}(H)} \leq \frac{M^{2}}{\omega}, \quad \operatorname{Tr} R=\int_{0}^{\infty}\left\|e^{A t}\right\|_{\mathrm{HS}}^{2} d t
$$

we may arrive at more explicit forms of the assumption (1.19) in terms of the quantities (1.21) and the constants $K_{f}, K_{\sigma}$. In particular, if $f$ and $\sigma$ are bounded then

$$
-|y|^{2}+\langle R y, f(y)\rangle+\frac{1}{2} \operatorname{Tr}\left(R \sigma(y) \sigma^{*}(y)\right) \leq-|y|^{2}+k_{1}\|R\|_{\mathscr{L}(H)}|y|+k_{2} \operatorname{Tr} R
$$

for some constants $k_{1}, k_{2}$ and the right hand side is always negative for $y \notin B_{L}$ provided that $L$ is sufficiently large.

By [15], Theorems 1.2, 1.3, we know that if $H=\Upsilon$, the operators $\sigma(y)$, $y \in H$, are invertible and

$$
\sup _{y \in H}\left\|\sigma^{-1}(y)\right\|_{\mathscr{L}(H)}<\infty,
$$

then (1.17) defines a strong Feller and irreducible process. By Theorem 2 this process is also $b w$-strong Feller and Theorem 4 implies that there exists an invariant probability measure for (1.17) if (1.19) is satisfied.

\section{Proofs}

Proof of Theorem 1. We shall prove Theorem 1 in several steps.

$1^{\circ}$ First, let us note that $Q_{t}^{-1 / 2} e^{A t}$ is a compact operator in $\mathscr{L}(H)$. The hypothesis (1.3) implies $\operatorname{Ker} Q_{t}^{1 / 2}=\{0\}$; moreover, we claim that $\operatorname{Rng} Q_{s}^{1 / 2} \subseteq \operatorname{Rng} Q_{t}^{1 / 2}$ for all $0<s \leq t$. By [5], Theorem 1, it suffices to check that $\left|Q_{s}^{1 / 2} h\right| \leq\left|Q_{t}^{1 / 2} h\right|$ for every $h \in H$, but obviously

$$
\left|Q_{s}^{1 / 2} h\right|^{2}=\left\langle Q_{s} h, h\right\rangle=\int_{0}^{s}\left|\Sigma^{*} e^{A^{*} r} h\right|^{2} d r \leq \int_{0}^{t}\left|\Sigma^{*} e^{A^{*} r} h\right|^{2} d r=\left|Q_{t}^{1 / 2} h\right|^{2} .
$$

From (1.3) we know that $\operatorname{Rng} e^{A t / 2} \subseteq \operatorname{Rng} Q_{t / 2}^{1 / 2} \subseteq \operatorname{Rng} Q_{t}^{1 / 2}$, so $Q_{t}^{-1 / 2} e^{A t / 2}$ is a well defined linear operator on $H$, whose boundedness follows by the closed graph theorem. Since $Q_{t}^{1 / 2}$ is a Hilbert-Schmidt operator, $e^{A t / 2}$ is also a compact operator, again by (1.3). Therefore, $Q_{t}^{-1 / 2} e^{A t}=Q_{t}^{-1 / 2} e^{A t / 2}$ 。 $e^{A t / 2}$ is compact.

$2^{\circ}$ From now on, let $t>0$ and $y_{n}, y_{0} \in H, y_{n} \rightarrow y_{0}$ weakly, be arbitrary but fixed. For brevity, denote the centered Gaussian measure $\mathscr{N}\left(0, Q_{t}\right)$ on $H$ by $\mu$. The operator $Q_{t}$ is self-adjoint, positive and compact, thus there exist $\lambda_{k} \geq 0$ and an orthonormal basis $\left\{e_{k}\right\}_{k \geq 1}$ of $H$ such that $Q_{t} e_{k}=\lambda_{k} e_{k}$, 
$k \geq 1$. Recall that we have

$$
Q_{t}^{-1 / 2} z=\sum_{k=1}^{\infty} \frac{1}{\sqrt{\lambda_{k}}}\left\langle z, e_{k}\right\rangle e_{k}, \quad z \in \operatorname{Rng} Q_{t}^{1 / 2} .
$$

The series on the right hand side makes sense since $\left\langle z, e_{k}\right\rangle=0$ whenever $z \in \operatorname{Rng} Q_{t}^{1 / 2}$ and $\lambda_{k}=0$, and converges since $z \in \operatorname{Rng} Q_{t}^{1 / 2}$ if and only if

$$
\sum_{k=1}^{\infty} \frac{\left|\left\langle z, e_{k}\right\rangle\right|^{2}}{\lambda_{k}}<\infty
$$

As $e^{A t} y_{n} \in \operatorname{Rng} Q_{t}^{1 / 2}, n \geq 0$, the Feldman-Hájek theorem (see e.g. [9], Theorem 3.1) implies that the measures $\mathscr{N}\left(e^{A t} y_{n}, Q_{t}\right)$ and $\mu$ are equivalent and the Radon-Nikodym derivative is given by

$$
\frac{d \mathscr{N}\left(e^{A t} y_{n}, Q_{t}\right)}{d \mu}(x)=\exp \left(\left\langle Q_{t}^{-1 / 2} e^{A t} y_{n}, Q_{t}^{-1 / 2} x\right\rangle-\frac{1}{2}\left|Q_{t}^{-1 / 2} e^{A t} y_{n}\right|^{2}\right)
$$

for $\mu$-almost all $x \in H$. The meaning of the first term on the right hand side is obvious when $x \in \operatorname{Rng} Q_{t}^{1 / 2}$; in the general case we set

$$
\left\langle Q_{t}^{-1 / 2} e^{A t} y_{n}, Q_{t}^{-1 / 2} x\right\rangle=\sum_{k=1}^{\infty} \frac{\left\langle e^{A t} y_{n}, e_{k}\right\rangle\left\langle x, e_{k}\right\rangle}{\lambda_{k}}
$$

and it is known that this series converges in $L^{2}(\mu)$. For later use, let us recall the argument. For a fixed $n \geq 0$, set $\xi_{k}=\lambda_{k}^{-1}\left\langle e^{A t} y_{n}, e_{k}\right\rangle\left\langle\cdot, e_{k}\right\rangle$, then $\xi_{k}$ is a Gaussian random variable on the probability space $(H, \mathscr{B}, \mu)$ and

$$
\int_{H} \xi_{k} d \mu=0, \quad \int_{H} \xi_{k} \xi_{l} d \mu= \begin{cases}0, & k \neq l, \\ \frac{\left|\left\langle e^{A t} y_{n}, e_{k}\right\rangle\right|^{2}}{\lambda_{k}}, & k=l .\end{cases}
$$

In particular, the functions $\left\{\xi_{k}\right\}$ are independent and

$$
\sum_{k=1}^{\infty} \int_{H}\left|\xi_{k}\right|^{2} d \mu=\sum_{k=1}^{\infty} \frac{\left|\left\langle e^{A t} y_{n}, e_{k}\right\rangle\right|^{2}}{\lambda_{k}}=\left|Q_{t}^{-1 / 2} e^{A t} y_{n}\right|^{2}<\infty,
$$

so the series $(2.1)$ is convergent in $L^{2}(\mu)$ and $\mu$-almost surely. Denote its sum by $\psi_{n}, n \geq 0$; then $\psi_{n}$ is a Gaussian random variable on $(H, \mathscr{B}, \mu)$ with law $\mathscr{N}\left(0,\left|Q_{t}^{-1 / 2} e^{A t} y_{n}\right|^{2}\right)$.

$3^{\circ}$ Towards the proof of (1.4), let us note that (1.4) is equivalent to

$$
\lim _{n \rightarrow \infty} \frac{d R_{t}\left(y_{n}, \cdot\right)}{d \mu}=\frac{d R_{t}\left(y_{0}, \cdot\right)}{d \mu} \quad \text { in } L^{1}(\mu) .
$$

The sequence $\left\{y_{n}\right\}$ is weakly convergent (thus bounded) and $Q_{t}^{-1 / 2} e^{A t}$ is a compact operator. Hence

$$
\lim _{n \rightarrow \infty}\left|Q_{t}^{-1 / 2} e^{A t}\left(y_{n}-y_{0}\right)\right|=0,
$$


in particular

$$
\lim _{n \rightarrow \infty}\left|Q_{t}^{-1 / 2} e^{A t} y_{n}\right|^{2}=\left|Q_{t}^{-1 / 2} e^{A t} y_{0}\right|^{2}
$$

and there exists a constant $k_{0}<\infty$ such that

$$
\sup _{n \geq 0}\left|Q_{t}^{-1 / 2} e^{A t} y_{n}\right| \leq k_{0} \text {. }
$$

Repeating the considerations above we arrive at

$$
\begin{aligned}
\int_{H}\left|\psi_{n}-\psi_{0}\right|^{2} d \mu & =\int_{H}\left|\sum_{k=1}^{\infty} \frac{\left\langle e^{A t}\left(y_{n}-y_{0}\right), e_{k}\right\rangle}{\lambda_{k}}\left\langle\cdot, e_{k}\right\rangle\right|^{2} d \mu \\
& =\sum_{k=1}^{\infty} \frac{\left|\left\langle e^{A t}\left(y_{n}-y_{0}\right), e_{k}\right\rangle\right|^{2}}{\lambda_{k}} \\
& =\left|Q_{t}^{-1 / 2} e^{A t}\left(y_{n}-y_{0}\right)\right|^{2} \underset{n \rightarrow \infty}{\longrightarrow} 0,
\end{aligned}
$$

which together with (2.2) implies

$$
\exp \left(\psi_{n}-\frac{1}{2}\left|Q_{t}^{-1 / 2} e^{A t} y_{n}\right|^{2}\right) \underset{n \rightarrow \infty}{\stackrel{\mu}{\longrightarrow}} \exp \left(\psi_{0}-\frac{1}{2}\left|Q_{t}^{-1 / 2} e^{A t} y_{0}\right|^{2}\right) .
$$

Moreover, straightforward calculations show that

$$
\sup _{n \geq 0} \int_{H} \exp \left(p \psi_{n}\right) d \mu \leq \frac{1}{\sqrt{\pi}} \int_{-\infty}^{\infty} e^{\sqrt{2} k_{0} p s} e^{-s^{2}} d s<\infty
$$

for any $p>1$. So $\left\{\exp \left(\psi_{n}-\frac{1}{2}\left|Q_{t}^{-1 / 2} e^{A t} y_{n}\right|^{2}\right)\right\}_{n \geq 0}$ is a uniformly integrable set of functions and (2.3) yields

$$
\begin{aligned}
\lim _{n \rightarrow \infty} \int_{H} \mid & \exp \left(\psi_{n}-\frac{1}{2}\left|Q_{t}^{-1 / 2} e^{A t} y_{n}\right|^{2}\right) \\
& -\exp \left(\psi_{0}-\frac{1}{2}\left|Q_{t}^{-1 / 2} e^{A t} y_{0}\right|^{2}\right) \mid d \mu=0,
\end{aligned}
$$

which is the desired conclusion.

Proof of Theorem 3. First, fix an arbitrary $t>0$ and assume that (a)(d) are satisfied. Our goal is to prove that $y \mapsto P_{t}(y, \cdot)$ is a continuous function from $(H, b w)$ to the space of bounded measures on $\mathscr{B}$ equipped with the topology of setwise convergence. Equivalently, by [6], Lemma 3.15, we have to prove that, for any $y_{n}, y \in H$ such that $y_{n} \rightarrow y$ weakly, the set $\left\{P_{t}\left(y_{n}, \cdot\right): n \geq 1\right\}$ of measures is relatively compact in the topology of setwise convergence and

$$
\lim _{n \rightarrow \infty} \int_{H} f(z) P_{t}\left(y_{n}, d z\right)=\int_{H} f(z) P_{t}(y, d z) \quad \text { for all } f \in \mathscr{C}_{\mathrm{b}}(H, b w) .
$$


(Note that the quoted lemma from [6] is applicable since all Borel probability measures on $(H, b w)$ are Radon.) However, $(2.4)$ is just the bw-Feller property which holds by the assumption (c), and the relative compactness of $\left\{P_{t}\left(y_{n}, \cdot\right)\right\}$ is equivalent to equicontinuity: for all $B_{k} \in \mathscr{B}$ with $B_{k} \downarrow \emptyset$ we must prove

$$
\lim _{k \rightarrow \infty} \sup _{n \geq 1} P_{t}\left(y_{n}, B_{k}\right)=0 .
$$

To this end, fix a weakly convergent sequence $\left\{y_{n}\right\}$ in $H$ and $B_{k} \in \mathscr{B}$ with $B_{k} \downarrow \emptyset$. Since $R_{t}$ is $b w$-strong Feller by assumption,

$$
\lim _{k \rightarrow \infty} \sup _{n \geq 1} R_{t}\left(y_{n}, B_{k}\right)=0 .
$$

Define measures $\boldsymbol{Q}_{n}$ on $\mathscr{F}$ by $d \boldsymbol{Q}_{n}=U\left(y_{n}, t\right) d \boldsymbol{P}$. Using the Girsanov theorem we obtain

$$
\begin{aligned}
\sup _{n \geq 1} P_{t}\left(y_{n}, B_{k}\right)= & \sup _{n \geq 1} \boldsymbol{Q}_{n}\left\{Z^{y_{n}}(t) \in B_{k}\right\}=\sup _{n \geq 1} \int_{\Omega} \mathbf{1}_{B_{k}}\left(Z^{y_{n}}(t)\right) U\left(y_{n}, t\right) d \boldsymbol{P} \\
= & \sup _{n \geq 1}\left\{\int_{\left\{U\left(y_{n}, t\right)>L\right\}} \mathbf{1}_{B_{k}}\left(Z^{y_{n}}(t)\right) U\left(y_{n}, t\right) d \boldsymbol{P}\right. \\
& \left.+\int_{\left\{U\left(y_{n}, t\right) \leq L\right\}} \mathbf{1}_{B_{k}}\left(Z^{y_{n}}(t)\right) U\left(y_{n}, t\right) d \boldsymbol{P}\right\} \\
\leq & \sup _{n \geq 1} \int_{\left\{U\left(y_{n}, t\right)>L\right\}} U\left(y_{n}, t\right) d \boldsymbol{P}+L \sup _{n \geq 1} \boldsymbol{P}\left\{Z^{y_{n}}(t) \in B_{k}\right\} \\
= & \sup _{n \geq 1} \int_{\left\{U\left(y_{n}, t\right)>L\right\}} U\left(y_{n}, t\right) d \boldsymbol{P}+L \sup _{n \geq 1} R_{t}\left(y_{n}, B_{k}\right) .
\end{aligned}
$$

The first term goes to 0 as $L \rightarrow \infty$ by uniform integrability, the second one tends to 0 as $k \rightarrow \infty$ according to (2.5).

Further, assume that (a), (e), (f) are satisfied. As $\left(P_{t}\right)$ is strong Feller, it suffices to check that $P_{t}$ maps $\mathscr{C}_{\mathrm{b}}(H)$ to $\mathscr{C}_{\mathrm{b}}(H, b w)$. So, fix $t>0, y_{n}, y \in H$, $y_{n} \rightarrow y$ weakly, and $\varphi \in \mathscr{C}_{\mathrm{b}}(H)$, and note that the convergence in (1.9) holds in fact in $L^{1}(\boldsymbol{P})$ due to (a) and nonnegativity of $U$. Analogously, the convergence in (1.10) holds in $L^{1}(\boldsymbol{P})$ by the dominated convergence theorem. Proceeding as above we get

$$
\begin{aligned}
\left|P_{t} \varphi\left(y_{n}\right)-P_{t} \varphi(y)\right|= & \left|\int_{\Omega} \varphi\left(Z^{y_{n}}(t)\right) U\left(y_{n}, t\right) d \boldsymbol{P}-\int_{\Omega} \varphi\left(Z^{y}(t)\right) U(y, t) d \boldsymbol{P}\right| \\
\leq & \left|\int_{\Omega} \varphi\left(Z^{y_{n}}(t)\right)\left\{U\left(y_{n}, t\right)-U(y, t)\right\} d \boldsymbol{P}\right| \\
& +\left|\int_{\Omega} U(y, t)\left\{\varphi\left(Z^{y_{n}}(t)\right)-\varphi\left(Z^{y}(t)\right)\right\} d \boldsymbol{P}\right|
\end{aligned}
$$




$$
\begin{gathered}
\leq \sup _{H}|\varphi| \int_{\Omega}\left|U\left(y_{n}, t\right)-U(y, t)\right| d \boldsymbol{P} \\
\quad+L \int_{\Omega}\left|\varphi\left(Z^{y_{n}}(t)\right)-\varphi\left(Z^{y}(t)\right)\right| d \boldsymbol{P} \\
\quad+2 \sup _{H}|\varphi| \int_{\{U(y, t)>L\}} U(y, t) d \boldsymbol{P}
\end{gathered}
$$

for any $L>0$, hence

$$
\lim _{n \rightarrow \infty} P_{t} \varphi\left(y_{n}\right)=P_{t} \varphi(y)
$$

follows easily. We see that $P_{t} \varphi$ is weakly sequentially continuous, and so bw-continuous.

Proof of Theorem 4. The statement (a) of the theorem may be proven in the same way as Lemma 5.1 in [16]. However, the proof in [16] is slightly flawed, so we use the opportunity to present here a corrected version. Let $\tau_{J}$ be the first hitting time of $J$, and define by induction

$$
\begin{aligned}
\mathfrak{t}_{1} & =\tau_{J} \\
\mathfrak{t}_{k+1} & =\inf \left\{t>\mathfrak{t}_{k}+2: X_{t} \in J\right\}=\mathfrak{t}_{k}+2+\theta_{\mathfrak{t}_{k}+2} \tau_{J}, \quad k \geq 1 .
\end{aligned}
$$

Since the set $J$ is recurrent and closed, we have $\mathfrak{t}_{k}<\infty$ and $X\left(\mathfrak{t}_{k}\right) \in J$ $\boldsymbol{P}_{x}$-almost surely for all $x \in H$ and $k \in \mathbb{N}$. Fix an arbitrary Borel set $B \in \mathscr{B}$ such that $P_{1}(0, B)>0$; again, we use $\tau_{B}$ to denote the first hitting time of $B$. Obviously,

$$
\boldsymbol{P}_{x}\left\{\tau_{B} \leq 1\right\} \geq P_{1 / 2}(x, B)>0
$$

for each $x$, thus

$$
\inf _{x \in J} \boldsymbol{P}_{x}\left\{\tau_{B} \leq 1\right\} \geq \inf _{x \in J} P_{1 / 2}(x, B) \equiv q>0
$$

due to the $b w$-compactness of $J$ and the $b w$-continuity of $P_{1 / 2}(\cdot, B)$. Set

$$
\mathfrak{d}_{k}=\inf \left\{t>\mathfrak{t}_{k}: X_{t} \in B\right\}, \quad k \geq 1 .
$$

Then

$$
\theta_{\mathfrak{t}_{k}}\left\{\tau_{B} \leq 1\right\}=\left\{\omega \in \Omega: \mathfrak{d}_{k} \leq \mathfrak{t}_{k}+1\right\}
$$

so $\theta_{\mathfrak{t}_{k}}\left\{\tau_{B} \leq 1\right\} \in \mathscr{F}_{\mathfrak{t}_{k}+1} \subseteq \mathscr{F}_{\mathfrak{t}_{k+1}}$ and using the strong Markov property we obtain

$$
\begin{aligned}
\sum_{k=1}^{\infty} \boldsymbol{P}_{x}\left(\theta_{\mathfrak{t}_{k}}\left\{\tau_{B} \leq 1\right\} \mid \mathscr{F}_{\mathfrak{t}_{k}}\right) & =\sum_{k=1}^{\infty} \boldsymbol{P}_{X\left(\mathfrak{t}_{k}\right)}\left\{\tau_{B} \leq 1\right\} \geq \sum_{k=1}^{\infty} \inf _{y \in J} \boldsymbol{P}_{y}\left\{\tau_{B} \leq 1\right\} \\
& \geq \sum_{k=1}^{\infty} q=+\infty \quad \boldsymbol{P}_{x} \text {-almost surely }
\end{aligned}
$$


for every $x \in H$. Therefore, the generalized Borel-Cantelli lemma (see e.g. [17], Corollary VII.5.2) implies that $\boldsymbol{P}_{x^{-}}$-almost every $\omega \in \Omega$ belongs to some $\theta_{\mathfrak{t}_{j}}\left\{\tau_{B} \leq 1\right\}$, and we have $\tau_{B}(\omega) \leq \mathfrak{d}_{j}(\omega) \leq \mathfrak{t}_{j}(\omega)+1<\infty$.

The statement (b) of Theorem 4 coincides with Proposition 5.9 of [16]. In [16], it is assumed that the state space is a separable metrizable Radon space, but in the proof of Proposition 5.9 the metrizability hypothesis is used only once (in the proof of Lemma 5.2) to ensure that the state space is Lindelöf. The space $(H, b w)$ is $\sigma$-compact, hence Lindelöf, and the argument from [16] may be used to prove existence of an invariant measure without alterations.

Proof of Theorem 5. Let $L \subseteq H$ be an arbitrary weakly compact set. Define

$$
u(x)=\int_{0}^{\infty} P_{t}(x, K) d t, \quad x \in H
$$

then $u \in \mathrm{b} \mathscr{B}$ by Theorem 2.1 in [16]. We claim that $u$ is bw-lower-semicontinuous: obviously, the functions $u_{N}=\int_{0}^{N} P_{t}(\cdot, K) d t$ are bw-continuous owing to the $b w$-strong Feller property and $u_{N} \nearrow u$ on $H$, so the set

$$
\{u>\alpha\}=\bigcup_{N=1}^{\infty}\left\{u_{N}>\alpha\right\}
$$

is $b w$-open for all $\alpha \in \mathbb{R}$. Further, since $P_{t}(x, K)>0$ for each $t>0$ and $x \in H$ we have $u>0$ on $H$ and $(\{u>1 / n\})_{n=1}^{\infty}$ is a nondecreasing sequence of $b w$-open sets covering the $b w$-compact set $L$, hence there exists $m \in \mathbb{N}$ such that $u>1 / m$ on $L$. Now we may conclude that $L$ is transient proceeding exactly in the same way as in [7], p. 403, or in [16], p. 298. For completeness, we repeat the easy argument. Fix $x \in H$, let $\tau_{L}$ be the first hitting time of $L$ and denote by $\tau(k)=k+\theta_{k} \tau_{L}$ the first hitting time of $L$ after $k, k \in \mathbb{N}$. We have $u(X(\tau(k)))>1 / m \boldsymbol{P}_{x}$-almost everywhere on the set $\{\tau(k)<\infty\}$, because $X(\tau(k)) \in L \boldsymbol{P}_{x}$-almost surely on $\{\tau(k)<\infty\}$, whence

$$
\begin{aligned}
\frac{1}{m} \boldsymbol{P}_{x}\{\tau(k)<\infty\} & \leq \boldsymbol{E}_{x} \mathbf{1}_{\{\tau(k)<\infty\}} u(X(\tau(k))) \\
& =\boldsymbol{E}_{x} \mathbf{1}_{\{\tau(k)<\infty\}} \int_{0}^{\infty} P_{r}(X(\tau(k)), K) d r \\
& =\boldsymbol{E}_{x} \mathbf{1}_{\{\tau(k)<\infty\}} \int_{0}^{\infty} \boldsymbol{P}_{x}\left(X(\tau(k)+r) \in K \mid \mathscr{F}_{\tau(k)}\right) d r
\end{aligned}
$$




$$
\begin{aligned}
& =\boldsymbol{E}_{x} \mathbf{1}_{\{\tau(k)<\infty\}} \int_{\tau(k)}^{\infty} \boldsymbol{P}_{x}\left(X(v) \in K \mid \mathscr{F}_{\tau(k)}\right) d v \\
& \leq \int_{k}^{\infty} \boldsymbol{P}_{x}\{X(v) \in K\} d v \\
& =\int_{k}^{\infty} P_{v}(x, K) d v \underset{n \rightarrow \infty}{\longrightarrow} 0
\end{aligned}
$$

by the strong Markov property. The sequence $(\{\tau(k)<\infty\})_{k=1}^{\infty}$ is nonincreasing, therefore for $\boldsymbol{P}_{x}$-almost all $\omega \in \Omega$ there exists $k$ satisfying $\tau(k)(\omega)$ $=\infty$, which is the desired conclusion.

\section{References}

[1] G. Da Prato and J. Zabczyk, Stochastic Equations in Infinite Dimensions, Cambridge Univ. Press, Cambridge, 1992.

[2] - - - Ergodicity for Infinite-Dimensional Systems, Cambridge Univ. Press, Cambridge, 1996.

[3] M. M. Day, Normed Linear Spaces, Springer, Berlin, 1958.

[4] C. Dellacherie and P.-A. Meyer, Probabilités et Potentiel. Chapitres IX à XI, Hermann, Paris, 1983.

[5] R. G. Douglas, On majorization, factorization, and range inclusion of operators on Hilbert space, Proc. Amer. Math. Soc. 17 (1966), 413-415.

[6] P. Gänssler, Compactness and sequential compactness in spaces of measures, Z. Wahrsch. Verw. Gebiete 17 (1971), 124-146.

[7] R. K. Getoor, Transience and recurrence of Markov processes, in: Séminaire de Probabilités XIV (Paris, 1978/1979), Lecture Notes in Math. 784, Springer, 1980, 397-409.

[8] I. V. Girsanov, Strongly Feller processes I. General properties, Teor. Veroyatnost. i Primenen. 5 (1960), 7-28 (in Russian).

[9] H.-H. Kuo, Gaussian Measures in Banach Spaces, Lecture Notes in Math. 463, Springer, Berlin, 1975.

[10] R. Liu, Ultimate boundedness and weak recurrence of stochastic evolution equations, Stochastic Anal. Appl. 17 (1999), 815-833.

[11] B. Maslowski, Stability of semilinear equations with boundary and pointwise noise, Ann. Scuola Norm. Sup. Pisa Cl. Sci. (4) 22 (1995), 55-93.

[12] B. Maslowski and J. Seidler, Probabilistic approach to the strong Feller property, Probab. Theory Related Fields 118 (2000), 187-210.

[13] - - - On sequentially weakly Feller solutions to SPDE's, Atti Accad. Naz. Lincei Cl. Sci. Fis. Mat. Natur. Rend. Lincei (9) Mat. Appl. 10 (1999), 69-78.

[14] R. E. Megginson, An Introduction to Banach Space Theory, Springer, New York, 1998.

[15] S. Peszat and J. Zabczyk, Strong Feller property and irreducibility for diffusions on Hilbert spaces, Ann. Probab. 23 (1995), 157-172.

[16] J. Seidler, Ergodic behaviour of stochastic parabolic equations, Czechoslovak Math. J. 47 (122) (1997), 277-316. 
[17] A. N. Shiryayev, Probability, Springer, New York, 1984.

[18] J. Zabczyk, Parabolic equations on Hilbert spaces, in: Stochastic PDE's and Kolmogorov Equations in Infinite Dimensions (Cetraro, 1998), G. Da Prato (ed.), Springer, Berlin, 1999, 117-213.

Mathematical Institute

Academy of Sciences

Žitná 25

11567 Praha 1, Czech Republic

E-mail: \{maslow, seidler\}@math.cas.cz

Received May 31, 2000

Revised version April 20, 2001 